\title{
Degradation of Communication Range in VANETs Caused by Interference 2.0 - Real-World Experiment
}

\author{
Robert K. Schmidt ${ }^{2}$, Bernhard Kloiber ${ }^{1}$, \\ Florian Schüttler ${ }^{2}$, and Thomas Strang ${ }^{1}$ \\ 1 Deutsches Zentrum für Luft- und Raumfahrt (DLR), \\ Münchner Strasse 20, 82234 Wessling, Germany \\ 2 DENSO AUTOMOTIVE Deutschland GmbH, \\ Freisinger Strasse 21, 85386 Eching, Germany
}

\begin{abstract}
High channel load in vehicle-to-vehicle communication leads to a degradation of the vehicles' communication range, due to interference and hence packet loss at larger distances. Packet loss results from two or more concurrent transmissions, colliding at receivers located inbetween, which is also known as the hidden station problem. In previous works, our simulation study has shown that this packet loss leads to a degradation of $90 \%$ of the communication range. In this paper, we confirm the simulation results by real-world measurements. We present a methodology for transferring the simulation scenario to a real-world measurement scenario, able to evaluate the problem of hidden stations. With three radios applying the IEEE 802.11 p standard, we measure the degradation of the communication range under interference. In the measurement, we find a degradation of 50 to $70 \%$. On the one hand, there are less collisions due to only one hidden station. On the other hand, we identify that the receiving vehicle as a shadowing object itself is an additional origin for hiding the other station which slightly increases the number of collisions even at close distances.
\end{abstract}

Keywords: Congestion, Field test, Interference, High load, Measurement, VANET, V2V.

\section{Introduction}

Enabling vehicles to communicate with each other spontaneously opens a new field for active safety applications. Vehicles equipped with wireless communication technologies according to IEEE 802.11p may exchange their status and movement. Such information on vehicles is included in Cooperative Awareness Messages (CAMs) which are periodically broadcasted to vehicles in the vicinity. All vehicles store the information in a local dynamic map of their surrounding [12. Thus, vehicles are able to check for potential dangerous situations like collisions at intersections or an approaching ambulance vehicle.

In high vehicle density like on a multi-lane highway, the periodicity of CAMs causes an overload of the communication channel. There, packet loss occurs due

T. Strang et al. (Eds.): Nets4Cars/Nets4Trains 2011, LNCS 6596, pp. 176-188, 2011.

(C) Springer-Verlag Berlin Heidelberg 2011 
to a high number of collisions in medium access. The well-known hidden station problem becomes more likely which, even worse, prevents the carrier sensing from working properly. We have analyzed the reasons and consequences of the channel overload by means of simulation. In [1] we found that in extreme situations the communication range under interference is reduced by $90 \%$. Successive packet loss and high load on the medium also causes a significant increase of the neighbor update delay 7 .

In this paper, we compare the simulation results with the results from the real-world experiment. We firstly provide a methodology on how to simplify and transfer the simulation scenario to a basic measurement scenario. We secondly conduct the measurement and evaluate the results, followed by a comparison to the results obtained from the simulation study. The presented scenario also forms the basis for testing countermeasures against packet loss.

The remainder of the paper is structured as follows. The next section reviews the literature related to measurements of wireless communication under high channel load. In Sec. 3, the methodology for our measurement is explained. Sec. 4 shows the results of the measurement, followed by the conclusions given in Sec. 5 .

\section{Related Work}

Weinfield describes in [3] a testbed for emulating multiple communicating vehicles. Using this testbed, he conducts experiments at different high channel load in an in-lab test. The testbed comprises 15 units each equipped with two IEEE $802.11 \mathrm{p}$ radios. All units are placed in a distance of 10 meters to each other. To emulate higher distances, different attenuators are attached to the radios. Both radios are used in order to emulate higher numbers of (virtual) vehicles, up to 180. In different measurements, the transmission-related parameters are varied, i.e. data rate of 6 and $12 \mathrm{MBit} / \mathrm{s}$, transmit power of 10 and $20 \mathrm{dBm}$, packet generation rate of 5 and $10 \mathrm{~Hz}$, message sizes of 300, 378 and 464 Bytes. The results show the highest increase of packet loss at the units with higher attenuation, i.e. higher emulated distance. However, in this test, the maximum attenuation is still too low to have a significant number of hidden stations as in our simulation study.

In [6] by Ramachandran et al., a measurement of the performance of many-tomany broadcast applications is conducted. A testbed of 400 small PCs with two transceivers is used for this experiment. The PCs are mounted at the ceiling of a big hall. Out of these $400 \mathrm{PCs}, 100$ radios are used at the same time to emulate a high dense vehicular network. The authors state that the results are valid only for this snapshot. Locations and hence distances between the radios are fixed. Also, the stations are close to each other so that no hidden stations occur. The experiment considers two workload scenarios: First, all stations saturate the medium by generating as much packets as can be actually transmitted. Second, all stations transmit 10 packets per second. As the authors conclude, with 100 radios and 10 packets per second they do not saturate the channel. Thus, the 
Table 1. Comparison of related work

\begin{tabular}{|l||c|c|c|}
\hline & Weinfield & Ramachadran et al. & Jardosh et al. \\
\hline \hline Protocol & IEEE $802.11 \mathrm{p}$ & IEEE $802.11 \mathrm{a}$ & IEEE $802.11 \mathrm{~b}$ \\
\hline Units physical/virt. & $15 / 180$ & $400 / 100$ & max. 523 / all \\
\hline Scenario & Lab-test & Big hall & Multi-room conference \\
\hline Communication & Broadcast & Broadcast (Ad-hoc) & Unicast (Infrastructure) \\
\hline Measured values & CBT, good packets & PDR, good packets & No. of frames, acceptance delay \\
\hline Workload & $5 / 10 \mathrm{~Hz}$ & $10 \mathrm{~Hz} /$ Saturation & typical Internet over WLAN \\
\hline Packet sizes & $300,378,464$ Byte & 128 Byte & $0-1200$ Byte \\
\hline Duration & $300 \mathrm{~s}$ & $120 \mathrm{~s}$ & $5: 33 \mathrm{~h} \& 2: 46 \mathrm{~h}$ \\
\hline Hidden stations & Few & None & Not stated, but probably many \\
\hline Spatial separation & Fixed & Fixed & Unknown \\
\hline
\end{tabular}

packet delivery rate stays above $95 \%$. However, for the saturated channel, the packet delivery rate goes down to $45 \%$. A proper countermeasure to mitigate this degradation is to enable the packet capturing feature.

Jardosh et al. 4 investigate nearly a full day of wireless LAN communication at a conference. Various channel loads and packet sizes have been logged and analyzed. They find that under high load, RTS-CTS cannot guarantee fair medium access and should be avoided. Higher data rates should be used to reduce the transmission time. However, it is difficult to compare the results of Jardosh et al. as they use IEEE 802.11 b in unicast mode with access point association.

To summarize and compare the related work, Tab. 1 highlights the differences between these experiments. All experiments mainly focus on in-lab tests and thus non-realistic channel conditions for vehicle-to-vehicle communication. The static experiments highlight the packet loss under high load, mostly using broadcast communication. However, due to the experiment setup only few hidden stations occur. No attention is paid to the variation of the spatial separation of the transceivers and their interrelation.

In our real-world experiment, we explicitly want to have all effects of signal propagation occurring outdoors, as this will be the case in the real-system on the road. We focus on the spatial separation of two stations potentially leading to hidden stations and provoking excessive packet loss at certain distances as found by our simulation study in [1].

\section{Methodology}

Following, we review the application scenario of the simulation study. Based on the road traffic scenario in the simulation, we explain the procedure to simplify and transfer the simulation scenario to the measurement scenario. Finally, we describe the hardware and software equipment being used for the measurement and its setup.

\subsection{Application Scenario}

For the simulation study in 1], we designed a scenario which involves many special communication aspects of vehicle-to-vehicle communication: High velocity, quickly changing vehicle density resulting in a severe hidden station scenario. 
Such a situation can easily happen when an ambulance vehicle (AV) approaches a slowly moving traffic jam, i.e. an area of high vehicle density. We analyze the packet receive ratio at the very end of the traffic jam, i.e. the tailend vehicle, with respect to the packets sent by the AV.

All vehicles apply a static (high) CAM rate. Due to the spatial separation, the transmissions of the vehicles within the traffic jam are partially hidden to the AV. Hence, there is a high likeliness of having two successive and interfering transmissions leading packet loss at the tail-end vehicles. In the end, the tail-end vehicles cannot receive the packets from the AV. We will review the simulation results when we compare them with the measurement results in Sec. 4.2 ,

\subsection{Scenario Transfer}

As real-world measurements usually involve an uncontrollable environment compared to the simulation, we carefully select the aspects that should be considered for the scenario transfer, guided by the following questions:

1. What are the most significant factors and aspects that cause the degradation and hence need to be transfered?

2. What can be eliminated from the simulation scenario to have a more controllable environment?

Transfer - Most important for the transfer is the variation of the spatial separation of the transmitter to receiver and interferer. We selected a sufficiently large scenario at a private airport next to DLR in Oberpfaffenhofen (Munich). It offers a 2286 x 45 meter runway mainly in free-space environment with nearly no reflecting objects. Most hangars are covered with grass which will scatter the signals rather than reflect them. We transfered the spatial separation as shown in Fig. 1. Similar to the simulation, we fixed the receiving vehicle's location (light grey circles, R3 ... R1) where the measurements log files are created. As a high

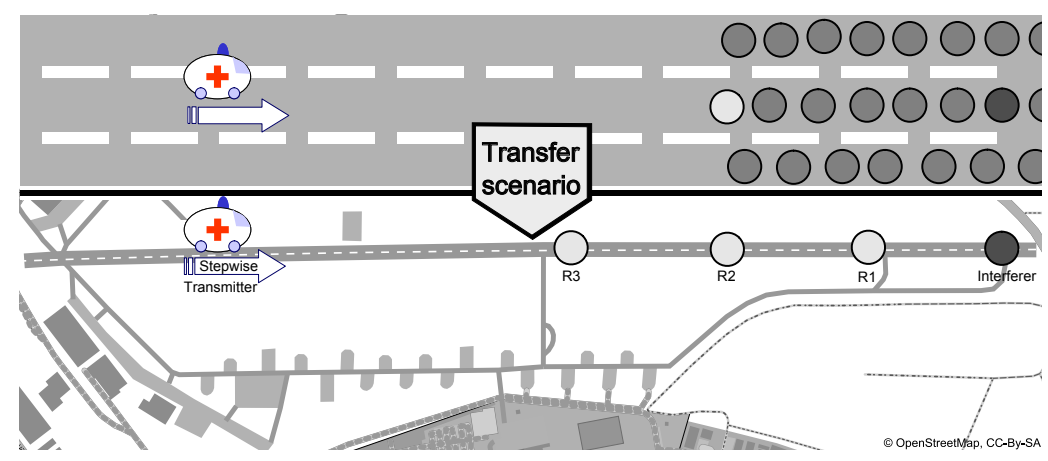

Fig. 1. Sketch of the spatial scenario transfer from simulation to real-world 
number of interfering vehicles is very difficult to setup and control, we decided to let one vehicle fully utilize the channel (dark grey circle). This interfering vehicle $I$ generates as much packets as actually can be transmitted with respect to the MAC protocol. With this high load, it is expected to get the same result of communication range degradation as for the highest CAM rate, i.e. $90 \%$.

Compared to the simulation, it is very difficult to achieve the same communication range with different vehicles. Due to roof curvature, antenna type and antenna position, the achieved range can vary significantly. Hence, we conducted a rough calibration of the transmit power prior to the measurement to achieve the same communication range as in the simulation. Details on that will be described in the following subsection.

Elimination - Due to the difficulty to drive vehicles in a reproduceable manner, we decided to avoid movement while measuring. Measurements are done only when all vehicles are standing still. This also avoids different antenna gains while the vehicle is pitching, due to accelerating or decelerating. After each measurement, the transmitting vehicle $T$ is moved stepwise. The interfering vehicle always remains at the end of the runway. The receiver is moved after accomplishing all transmitter position. This is in line with the simulation setup, where no movement-dependent signal propagation effects like doppler shift are modeled. As the simulation results rely on the free-space model, reflections and multi-path effects are also eliminated from the real-world scenario.

\subsection{Communication Range Calibration}

To be able to compare the results of the measurements with the results of the simulations, the transmission ranges are calibrated to approximately $1000 \mathrm{~m}$ as in the simulation. Both, interferer and transmitter, are calibrated against the receiver. The interferer is placed at the end of the runway, the receiver is placed at a distance of $1000 \mathrm{~m}$ to the interferer. Next, the transmission power of the interferer is adjusted, so that the receive ratio at the receiver is approximately $90 \%$ which we assume as the edge of the communication range. The same procedure is done for transmitter and receiver. As a result, the transmitter applies $18 \mathrm{dBm}$, the interferer applies $12 \mathrm{dBm}$. The significant difference between the calibration results is supposed to be due to antenna characteristics, roof curvature and elevation angle differences. Fig. 2 depicts the elevation profile 1 of the runway which poses slight angle differences in the elevation angle.

\subsection{Equipment and Setup}

Three cars have been used for the experiment, each equipped with the hardware and software as described in the following.

\footnotetext{
${ }^{1}$ The elevation profile of the runway has been created with a barometer-equipped GPS receiver.
} 


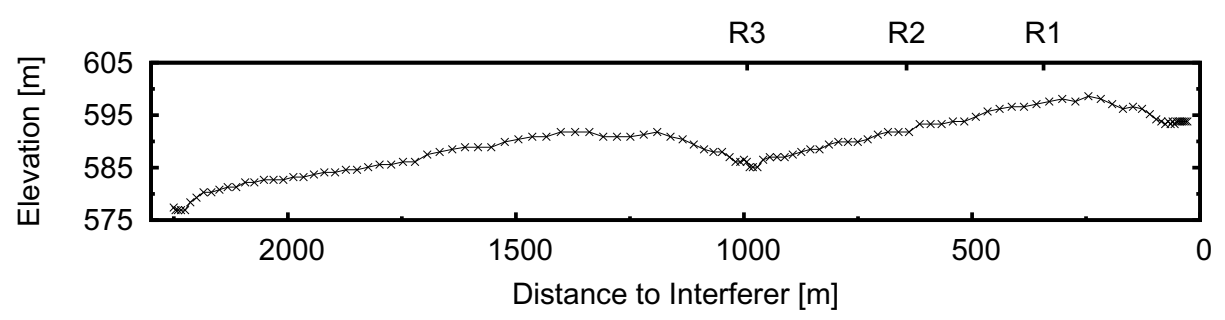

Fig. 2. Elevation profile of the runway

- Communication unit: The basic component is the WSU (Wireless Safety Unit) [2, which is an integrated prototype platform implementing the IEEE 802.11p standard [9]. All log data is written to a compact flash card installed at the receiving vehicle.

- Antenna: An antenna has been connected to each WSU by using a $3 \mathrm{~m}$ long antenna cable. The antennas have been mounted centered on the roof of each car. The transmitting and the interfering car have used a monopole antenna by Nippon Antenna having a gain of $0 \mathrm{dBi}$ in the horizontal and 5 $\mathrm{dBi}$ average gain in an elevation of $15^{\circ}$. The receiving car has been equipped with a dipole antenna by Mobile Mark which has a gain of $9 \mathrm{~dB} 2$ in omni direction.

- Positioning: Each WSU is connected with a Garmin GPS18-LVC receiver. The GPS receivers have been placed outside of line-of-sight to the communication direction, i.e. not in the center line of the roof.

- Logging: The test application used for the measurements is the WSU Test Application (WTA) [2]. It enables the user to test the performance of the communication channel and the MAC protocol. Furthermore, it configures the radio parameters, executes packet tests, displays real-time statistics, and logs key metrics. A screenshot of the GUI is shown in Fig. 3 .

- Vehicles: Transmitter and interferer vehicles are compact cars with a round roof curvature. The receiver vehicle is a big offroad vehicle with a flat roof.

Using the WTA, the radio is configured as summarized in Tab. 2, The data rate is set to $6 \mathrm{Mbit} / \mathrm{s}$ which was found to be optimal for VANETs [10]. The transmitter is configured to send 10 packets per second which is a usual message rate. By using the continuous mode of the WTA, the resulting channel busy time at the interferer is approximately $84-86 \%$.

As shown in Fig. 1, measurements are done at three different receiver locations, i.e. distances $R-I$ of $1000 \mathrm{~m}$ (R3), $650 \mathrm{~m}$ (R2) and $350 \mathrm{~m}$ (R1). For each receiver position, the transmitter starts at a distance of $900 \mathrm{~m}$ to the receiver and approaches in steps of $50 \mathrm{~m}$. We did not start the measurement at the calibrated communication range to eliminate the fluctuations of packet loss at the border of the communication range. For each step, the receiver logs the packets for 5

${ }^{2}$ Due to the flat roof of the receiving vehicle, we used a high gain bar antenna. 


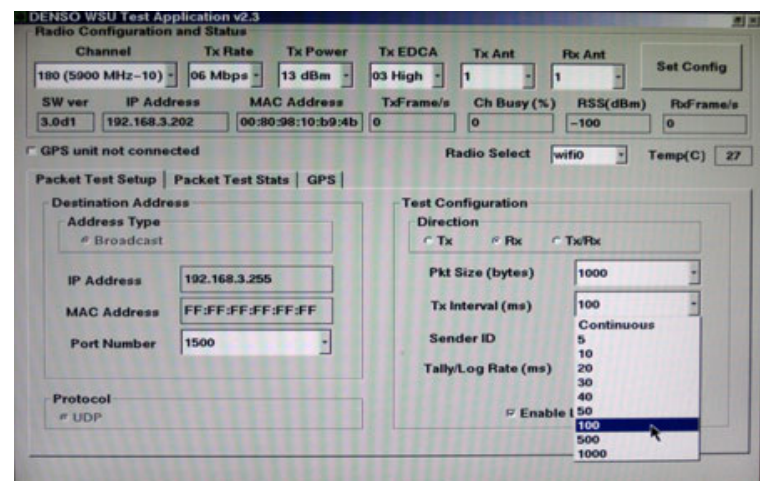

Fig. 3. Screenshot of the WTA showing the configuration of radio and packet test parameters

Table 2. Overview of the measurement parameters

\begin{tabular}{|l|r|}
\hline Fixed Parameter & Value \\
\hline Number of vehicles & 3 \\
\hline Channel & $180(5900 \mathrm{GHz})$ \\
\hline Receiver diversity & $\mathrm{Off}$ \\
\hline Transmit power (Transmitter/Interferer) & $\approx 12 \mathrm{dBm}$ \\
\hline Maximum communication range & $6 \mathrm{MBit} / \mathrm{s}$ \\
\hline Data rate & 1000 Bytes \\
\hline Packet length (payload) & 5 seconds \\
\hline Packet rate (Transmitter / Interferer) & $0 / 5 \mathrm{dBi}$ \\
\hline Measurement length & $9 \mathrm{dBi}$ \\
\hline Number of runs & Values \\
\hline Antenna gain - Nippon Antenna $\left(0^{\circ} / 15^{\circ}\right)$ & true, false \\
\hline Antenna gain - Mobile Mark & Continuous \\
\hline \hline Varied Parameters & $1000 \mathrm{~m}, 650 \mathrm{~m}, 350 \mathrm{~m}$ \\
\hline Interferer enabled & $900,850, \ldots, 50 \mathrm{~m}$ \\
\hline Distance $R-I$ & $R$ \\
\hline Distance $T$ - $R$ &
\end{tabular}

seconds. The logging procedure for each step is done twice, once with interferer switched off and once with interferer switched on. After the first run (i.e. one complete approach of the transmitter to the receiver for each receiver position) is finished, a second run is started with the same configuration parameters.

\section{Measurement Results and Comparison}

The results of the measurements are shown for each receiver location separately and afterwards summarized. In the last subsection, the trends of the communication range degradation are compared between the measurement and the simulation. 


\subsection{Measurement}

In the following, we present the results for the packet receive ratio at the receiver from the transmitter. The average values per run are connected with lines for the sake of readability. Note that the lines do not represent actual measurements. Single crosses or stars indicate single results for the packet receive ratio, i.e. the number of received packets per one second divided by the number of transmitted packets by the transmitter.

It is expected that the number of collisions drops significantly when the transmitter is closer to the receiver than the interferer so that the signal-tointerference-ratio (SIR), i.e. T's signal strength over I's signal strength, is sufficiently high. The packet capturing feature also allows to recover the stronger one from two colliding transmissions, even if it arrived after the reception of the weaker signal has already been started.

Receiver-Interferer distance 1000 meters - R3. When receiver and interferer are at the edge of communication range to each other, the received signal strength of the interferer is quite low. As the transmitter approaches the receiver at a distance of 600 meters, a first increase above one packet per second on average can be seen in Fig. 4. At 500 meters, the packet receive ratio is within a transient area. The SIR of both signals is in the order of the one needed at the receiver. $100 \%$ of the transmitted packets are received at the receiver at distance 450 meters. This remains nearly stable as the transmitter gets closer, except for single outliers at 350 and 200 meters.

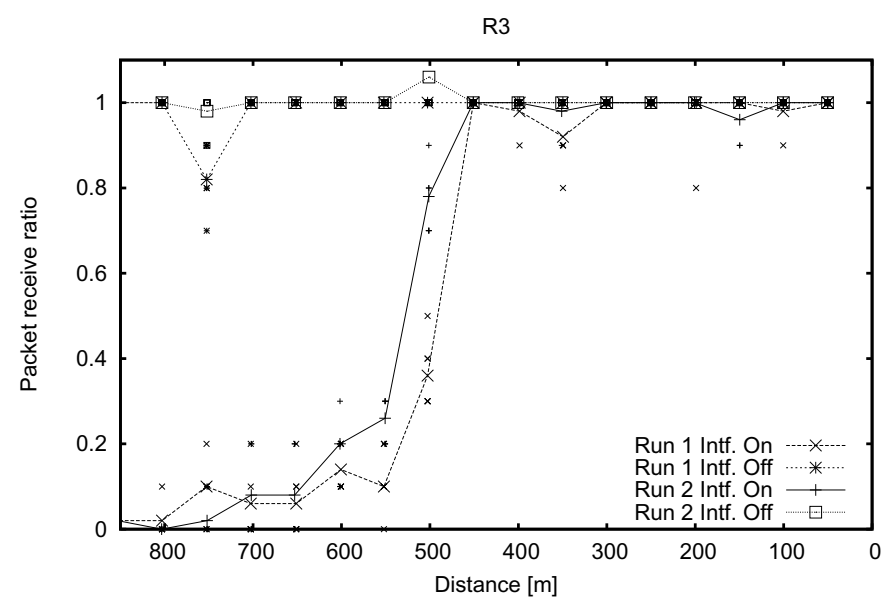

Fig. 4. Packet receive ratio - 1000 meters

Receiver-Interferer distance 650 meters - R2. At R2, receiver and interferer are within communication range to each other. Compared to R3, the results in Fig. [5 present that the transmitter needs to approach the receiver closer to be within communication range under interference. The transient area starts at 


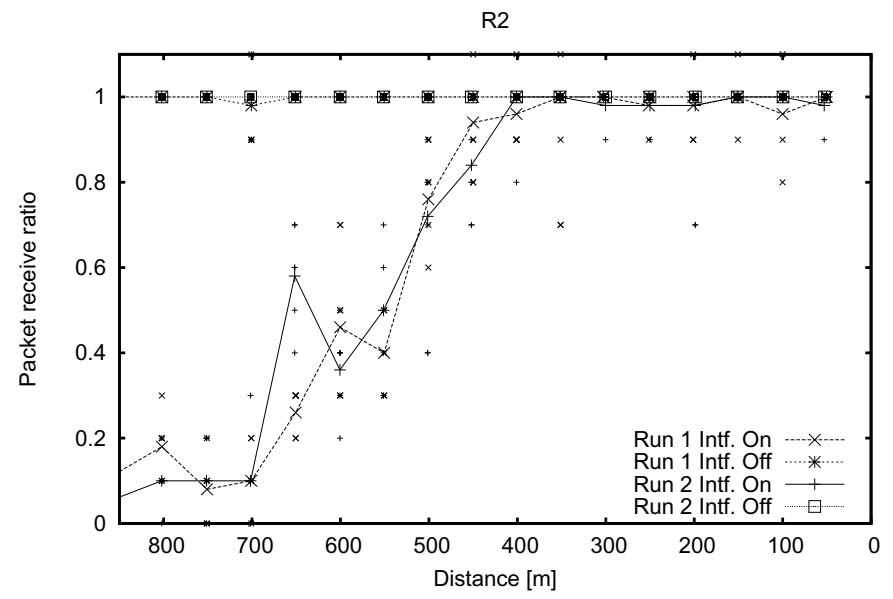

Fig. 5. Packet receive ratio - 650 meters

around 700 meters and stretches till 400 meters. Similar to R3, there are also some (more) outliers located at 350 and 200 meters. As receiver diversity is disabled, these similar results could be due to the ground reflection, canceling the signals at certain distances (i.e. following the two-ray-ground model).

Receiver-Interferer distance 350 meters - R1. The results for R1 depicted in Fig. 6 exhibit a much larger transient area of packet loss. The average value of the receive ratio is fluctuating much more and does never reach $100 \%$. As the signal strength of the interferer is relatively high at R1 compared to R2 and R3, the impact of the interferer is much more significant. As in R3 and R2, we also see outliers at 350 meters. After reviewing the simulation results in the following subsection, we will summarize the results for all receiver locations and consider the measurement for the channel busy time which will reveal more details about the above mentioned fluctuation.

Due to difficult weather conditions and increased air traffic, the measurements had been stopped after the first run of R1. However, compared to the other receiver locations, we assume to have a valid measurement for the receiver location R1.

\subsection{Simulation}

Fig. 7 depicts the simulation results published in [1]. All vehicles periodically broadcast packets of size 1000 Bytes. The message rates in the plot are varied 15, 18, 20 and $40 \mathrm{~Hz}$. For the sake of readability, these rates are selected for visualization as they show the low load, medium load and heavy overload on the communication channel. As seen in the figure, the packet receive ratio decreases when the message rate is increased from 15 to $18 \mathrm{~Hz}$, especially at larger 


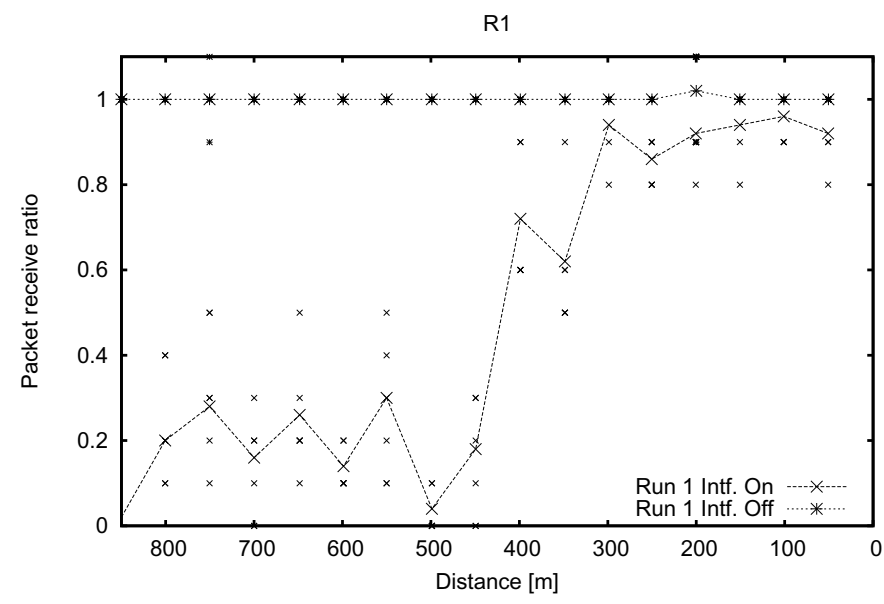

Fig. 6. Packet receive ratio - 350 meters

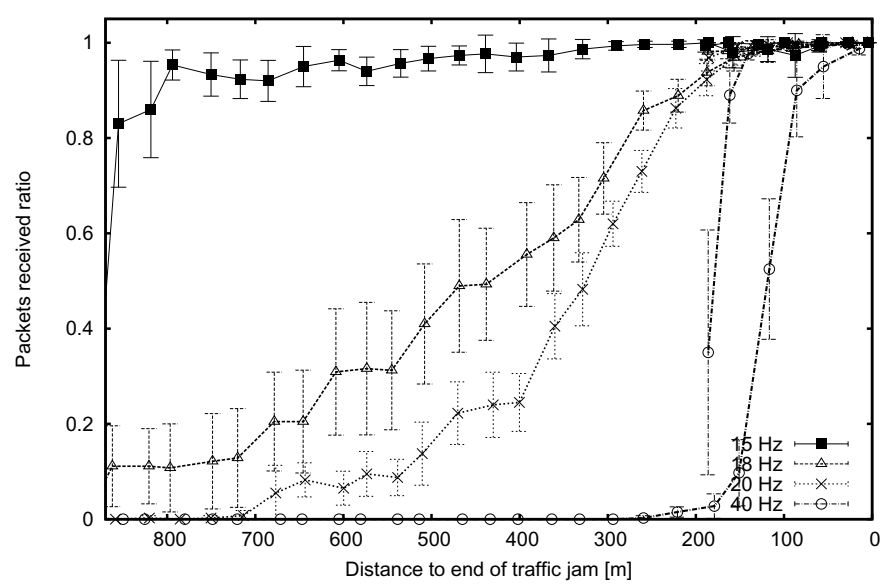

Fig. 7. Simulation result - Packet receive ratio depending on message rate and distance of approaching vehicle and tail-end vehicle

distances between tail-end vehicle and the ambulance vehicle. However, with further increase of the CAM rate $(40 \mathrm{~Hz})$ it converges, presenting a steep increase of the packet receive ratio at a distance of 100 meters. Hence, the communication range under interference is about $10 \%$ of the original communication range.

Summary and Comparison. At each receiver location, we have seen a significant degradation of the communication range between the transmitting vehicle and the receiving vehicle. To summarize and better understand the results, we also consider the interaction between the transmitting vehicle and the interfering vehicle with respect to the MAC protocol. Assuming roughly symmetric 


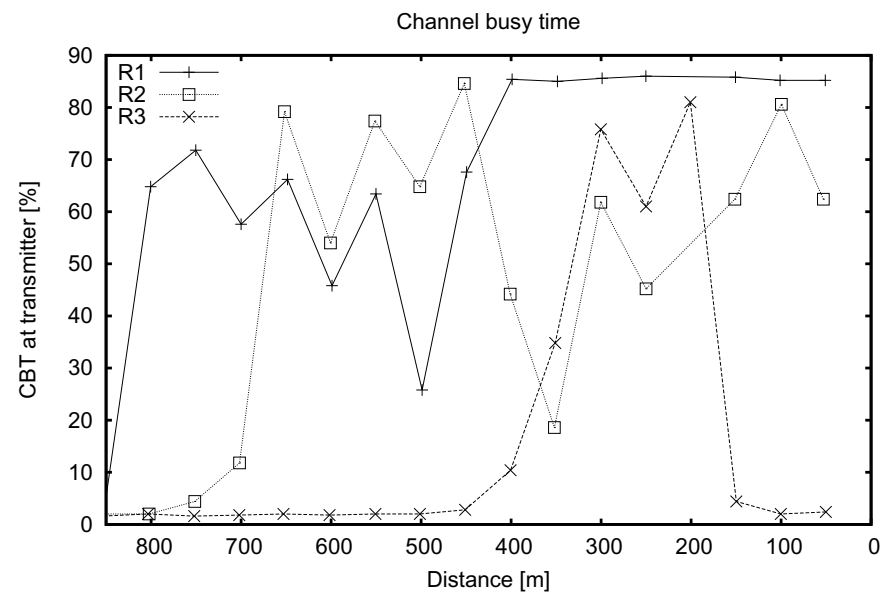

Fig. 8. Channel busy time at transmitter when interferer is flooding the channel

signal propagation between them, we can compare the results with the Channel Busy Time (CBT) at the transmitting vehicle when the interferer is switched on, shown in Fig. 8 .

The graphs of the CBT provides two aspects:

1. Synchronization of medium access between transmitter and interferer

2. Shadowing by the receiving vehicle.

The receive ratio at $\mathrm{R} 1$ increases at around 400 to 300 meters to the receiver. Adding up the distance between receiver and interferer roughly equals to the calibrated communication range. From this distance on, the MAC protocol has a chance to sense the transmissions of the interferer and avoid from medium access. At the same time, the interferer also senses the transmissions of the transmitter and also refrains from medium access so that the the distributed medium access protocol is able to reduce the number of collisions.

Similar trends are observable for R2 and R3. The graph for R2 increases at around 650 to 550 meters, for R3 the increase is at around 800 to 700 meters. However, following the graphs from left to right present significant dips. During the measurements, we log the distance where line-of-sight is significantly blocked by the receiving vehicle. The values are $\approx 150 \mathrm{~m}$ for $\mathrm{R} 3, \approx 300 \mathrm{~m}$, and $\approx 700 \mathrm{~m}$ for R1. For R1, this confirms the dips at 600 and 500 meters. Beyond that, the CBT goes to maximum which means that the signal attenuation due to shadowing is not significant. The transmitter is already quite close to the interferer. R2 has an even worse dip at 400 and 350 meters, followed by scattering results around $50 \% \mathrm{CBT}$. The worst shadowing is experienced for R3. The CBT drops much below $10 \%$ due to the shadowing. The signal is already strongly 
attenuated due to the large distance between interferer and receiver. Shadowing typically adds up 10 to $20 \mathrm{~dB}$ which then makes it impossible to sense any transmission at this distance.

When we now look back to the packet receive ratio, lower ability to sense packets due to shadowing has only few impact at lower distances as the SIR is sufficiently high. The graph in R3 has the same steep increase as in the simulation. For R2, the increase of the receive ratio is less steep. Some single measurements show a loss of $10 \%$ at lower distances (400 to 50 meters). Most of the fluctuation in the results is seen in the graph of R1. In this case, the better signal-to-interference ratio is an advantage for the interferer, especially due to packet capturing, more packets of the transmitter get lost.

The measurement showed a lower degradation than the extreme case in the simulation. Although the channel load is at maximum in both cases, the (reduced) number of interfering vehicles has a strong impact on the degradation. In the simulation, we found that the carrier sensing is not working properly in extreme high load situations. As the measurement comprises only three vehicles, the carrier sensing is mainly interfered by one hidden station at a time. The problem of many hidden stations leading to multiple interfering signals is not accounted in the measurement scenario.

The hidden stations are more wide spread in the simulation while they are isolated in the measurement. Hence the less steep increase in the transient area.

\section{Conclusions and Outlook}

Active safety applications in VANETs rely on receiving updates from neighboring vehicles frequently. Under high channel load, the packet error rate can significantly increase at higher distances between receiver and transmitter. In our previous work [1], we analyzed and quantified this degraded communication range due to interference.

We confirm in this paper the trend of communication range degradation under interference by a real-world experiment with a basic measurement setup of three vehicles. As a result, we already determine a degradation of $50 \%$ to $70 \%$. We further find that due to shadowing of the vehicles itself, additional packet loss occurs. The other transmitter becomes hidden due to significant signal attenuation in the shadow of the bigger vehicle. While in this shadow, another vehicle cannot correctly sense that the channel is busy.

The advantage in signal-to-interference ratio is supported by packet capturing which improves the communication range under interference. All in all, even under high channel load the communication range under interference is sufficiently large for time-critical safety applications operating in a range of 50 - 300 meters. No significant degradation of the packet receive ratio is found in this area.

As a next step, we plan to employ multiple interfering vehicles to be placed in the field, having a high CAM rate instead of flooding the channel. For our future work, we also plan to use the presented scenario as a basic validation scenario for Decentralized Congestion Control [8]. 


\section{Acknowledgements}

The authors would like to thank EDMO-Flugbetrieb GmbH [11] for the opportunity to run the experiments under almost ideal radio propagation conditions and for their safe coordination of accessing the runway alternating with normal air traffic.

\section{References}

1. Schmidt, R.K., Köllmer, T., Leinmüller, T., Böddeker, B., Schaefer, G.: Degradation of Transmission Range in VANETs caused by Interference, Praxis der Informationsverarbeitung und Kommunikation (PIK). Special Issue on Mobile Ad-hoc Networks 4 (2009)

2. Weinfield, A., Graham, S.: Methods to Reduce DSRC Channel Congestion and Improve V2V Communication Reliability. In: ITS World Congress, Busan, Korea (2010)

3. Weinfield, A.: Wireless Vehicular Safety Systems: Specialized Test Platform Supports Application Evaluation. In: ITS World Congress, Beijing, China (2007)

4. Jardosh, A.P., Ramachandran, K.N., Almeroth, K.C., Beldingroyer, E.M.: Understanding congestion in IEEE 802.11b wireless networks. In: Proceedings of the 2005 Internet Measurement Conference (2005)

5. Thorpe, C., Murphy, S., Murphy, L.: Analysis of Variation in IEEE802.11k Channel Load Measurements for Neighbouring WLAN Systems. In: ICT Mobile and Wireless Communications Summit (ICT-MobileSummit 2008), Stockholm, Sweden, June 10-12 (2008)

6. Ramachandran, K., Gruteser, M., Onishi, R., Hikita, T.: Experimental Analysis of Broadcast Reliability in Dense Vehicular Networks. In: VTC Fall (2007)

7. Kloiber, B., Strang, T., de Ponte-Müller, F., Garcia, C.R., Röckl, M.: An Approach for Performance Analysis of ETSI ITS-G5A MAC for Safety Applications. In: Proceedings of the The 10th International Conference on Intelligent Transport Systems Telecommunications (ITST), Kyoto, Japan (2010)

8. Schmidt, R.K., Brakemeier, A., Böddeker, B., Schäfer, G.: Architecture for Decentralized Mitigation of Local Congestion in VANETs. In: Proceedings of the The 10th International Conference on Intelligent Transport Systems Telecommunications (ITST), Kyoto, Japan (2010)

9. Institute of Electrical and Electronics Engineers, IEEEP802.11p/D9.0 Wireless Access in Vehicular Environments, Draft 9.0 (November 2009)

10. Maurer, J., Fugen, T., Wiesbeck, W.: Physical Layer Simulations of IEEE802.11a for Vehicle-to-Vehicle Communications. In: Proceedings of the 62nd IEEE Vehicular Technology Conference, VTC 2005 (2005)

11. EDMO-Flugbetrieb GmbH, http://www.edmo-airport.de/index.html

12. SAFESPOT, Deliverable D3.3.3 - Local dynamic map specification, Technical Report (2008) 